\title{
The dynamics of disordered materials studied by inelastic x-ray scattering
}

\author{
Francesco Sette $e^{1}$ and Giancarlo Ruocco ${ }^{2}$, \\ ${ }^{1}$ European Synchrotron Radiation Facility, Grenoble, France \\ ${ }^{2}$ Universitá di L'Aquila and Istituto Nazionale di Fisica della Materia, L'Aquila, Italy
}

is particularly relevant because it reflects the properties of the particle-particle correlation function at distances approaching the inter-particle distances and at times typical of density fluctuations at the interparticle level, i.e. in the pico-second range. This dynamics can be investigated by the experimental determination of the dynamic structure factor $S(Q, E)$ - the time and space Fourier transform of the particle-particle correlation function - in the momentum $(\mathrm{Q})$-energy $(\mathrm{E})$ regions of 0.1 to $20 \mathrm{meV}$ and 0.01 to $10 \mathrm{~nm}-1$.

In crystalline systems, this is one of the most successful applications of inelastic neutron scattering (INS) [1]. In disordered systems, the neutron technique has been successfully used to investigate the dynamics at momentum transfers typically larger than $10 \mathrm{~nm}-1$ [2]. At wavelengths longer and comparable to $d$, due to the kinematics conditions of available neutron spectrometers, serious difficulties are found to probe acoustic excitations with speeds of sound, v, larger than v@1500 m/s. The typical values of $v$ in liquids and glasses are either comparable or considerably larger than $1500 \mathrm{~m} / \mathrm{s}$, and this is the main reason why, in disordered systems, a comprehensive experimental picture of the high frequency collective dynamics is still missing.

Inelastic x-rays scattering (IXS) with $10 \pi 20 \mathrm{keV}$ incident energy is, in principle, a complementary method to INS for studying acoustic modes at small momentum transfers. In fact, providing sufficient energy resolution and incident beam intensity, one can access the desired phase space.

The IXS technique is a recent development, made possible by the high spectral flux density of the new synchrotron radiation sources, and by new ideas in $\mathrm{x}$-ray 
optics using Bragg diffraction from perfect crystals. This combination has allowed the construction of a unique IXS spectrometer, now in routine operation at the European Synchrotron Radiation $\mathrm{Fa}$ cility in Grenoble. Its main success lies in its capability to measure $S(Q, E)$ at momentum transfers, $\mathrm{Q}=0.5 \pi 30 \mathrm{~nm}-1$, with basically unlimited energy transfer, and with an energy resolution of $1.5 \pm 0.2 \mathrm{meV}$.

Thanks to the new instrument, it has been possible to begin to address issues on the high frequency dynamics of disordered materials as the following ones [3]: 1. Collective excitations have been discovered in all the investigated liquids and glasses at wavelengths approaching $\mathrm{d}$. The Q-dependence of their energy values testify that they are the short wavelength evolution of the hydrodynamic sound mode. Important deviations are observed, however, from phonons in crystals. In particular, their Q-dependent broadening suggests that these modes deviate from the plane waves found in crystals. At present one is trying to relate these deviations to the behavior of the sound excitations observed in the long wavelength limit, and, in glasses, to the anomalous specific heat and thermal conductivity found at low temperatures. In this respect, one would like to understand, when compared to the Debye behavior of the corresponding crystal, the origin of the excess specific heat at low temperature and the excess density of vibrational states found in glasses.

2. The interference between density fluctuations and microscopic structural relaxation processes have been clearly identified in glass-forming liquids as glycerol, ortho-terphenyl and in hydrogen bonded liquids as water. These studies have allowed determining experimentally, as a function of temperature and Q-transfer, important parameters as the infinte-frequency sound velocities. These parameters enters in the modeling of the $S(Q, E)$ of a liquid, and in the current efforts to relate the liquid-glass transition to a change in the ergodicity of the system [4].

The possibility to contribute with an experimental method to the understanding of such problems is creating a worldwide interest towards the IXS technique, and important results have been already documented [3]. In this respect, in fact, it is quite reasonable to expect important contributions not only on the high frequency dynamics of liquid systems, but also on glassy materials, on supercritical fluids, on complex chemical aggregates, on systems of biological interest, and more generally, to understand better the collective dynamics of disordered materials.

\section{References:}

[1]S.W. Lovesey, “Theory of neutron scattering from condensed matter", Clarendon Press, Oxford, 1984.

[2] U. Buchenau, H.M. Zhou, N. Nucker, K.S. Gilroy and W.A. Phillips, Phys. Rev. Lett. 60, 1318, (1988).

[3] F. Sette, G. Ruocco, M. Krisch, C. Masciovecchio and G. Monaco, Science 280 , 1550 (1998) and references therein.

[4] G. Parisi, Phys. Rev. Lett. 79, 3660 (1997). 